63 $3^{\text {ème }}$ Congrès de la SFCO, 03011 (2015)

DOI:10.1051/sfco/20156303011

(C) Owned by the authors, published by EDP Sciences, 2015

\title{
Résorptions des incisives latérales associées aux canines maxillaires incluses : intérêt diagnostic du CBCT
}

\author{
Bahi-Gross S*, Amar D*, Quinque E*, Bolender Y**, Feki A*, Gros C-L**** \\ * CHRU Strasbourg, UF Chirurgie Buccale, 1 place de l'Hôpital, 67000 Strasbourg \\ ** CHRU Strasbourg, UF Orthopédie Dento-Faciale, 1 place de l'Hôpital, 67000 Strasbourg \\ *** CHRU Strasbourg, UF Radiologie, 1 place de l'Hôpital, 67000 Strasbourg
}

Avec une prévalence évaluée entre 1 et $3 \%$, les canines maxillaires incluses sont en terme de fréquence en seconde position derrière les dents de sagesse (Walker 2004). Ces inclusions d'étiologie multiples peuvent avoir des conséquences fonctionnelles et esthétiques non négligeables.

Parmi ces conséquences, les résorptions des dents adjacentes et plus particulièrement des incisives latérales sont évoqués (Oberoi 2012).

Ces complications plus ou moins sévères sont évaluées à $30 \%$ en moyenne en fonction des études (Ericson 2000). Ces résorptions sont généralement peu diagnostiquées au cours des examens conventionnels (orthopantomogramme et radiographies rétro- alvéolaires).

Dans ce cadre et afin de limiter l'étendue de ces résorptions, des examens complémentaires type CBCT trouvent tout leur intérêt (Becker 2010).

Une question se pose : quand programmer au mieux cet examen complémentaire

- en début de traitement orthodontique avant la pose des multi-attaches

- en cours de traitement orthodontique avant le dégagement chirurgicoorthodontique.

Méthode

Une étude portant sur l'analyse rétrospective de 64 examens CBCT a été réalisée. Ces examens nécessaires dans le cadre du diagnostic de canines incluses maxillaires étaient programmés soit en début soit en cours de traitement orthodontique. Pour chacune des 83 canines maxillaires incluses, plusieurs éléments évocateurs ont été répertoriés : âge, sexe, position des dent incluses dans les 3 plans, présence ou non de résorptions des incisives latérales, étendue des résorptions, présence de multi- attaches...

Résultats

Il ressort de cette analyse que la localisation principale des canines maxillaires est palatine (53\%) et médiale. La proportion de résorptions des incisives latérales est évaluée à $25 \%$ : $19 \%$ présentant une résorption légère, $23 \%$ une résorption modérée et $57 \%$ une résorption sévère. Ces résorptions principalement localisées au niveau du tiers apical (66\%) et tiers moyen (33\%) des incisives latérales. Elles sont également plus fréquentes chez les patients appareillés et âgés de moins de 17 ans.

Conclusion

L'analyse de ces éléments permet de conclure que le CBCT représente un examen indispensable tant pour l'orthodontiste afin d'envisager la direction de traction de la dent incluse que pour le chirurgien oral permettant un abord chirurgical et un collage dans des conditions optimales (Lai 2013). L'examen par CBCT devrait donc être un examen complémentaire quasi systématique programmé idéalement avant tout déplacement orthodontique de l'incisive latérale et effectué dans des conditions proches de l'intervention chirurgicale.

This is an Open Access article distributed under the terms of the Creative Commons Attribution License 4.0, which permits unrestricted use, distribution, and reproduction in any medium, provided the original work is properly cited. 
$63^{\text {ème }}$ Congrès de la SFCO

Nom et adresse du conférencier

Sophie BAHI-GROSS

Strasbourg, UF Chirurgie Buccale

1 place de l'Hôpital

67000 Strasbourg (France)

sophie.bahi@wanadoo.fr 